\title{
The diagnostic accuracy of magnetic resonance imaging in predicting pathologic complete response after neoadjuvant chemotherapy in patients with different molecular subtypes of breast cancer
}

\author{
Xinfeng Zhang ${ }^{1,2,3}$, Dandan Wang ${ }^{4}$, Zhuangkai Liu ${ }^{1,2}$, Zheng Wang ${ }^{5}$, Qiang Li $^{5}$, Hong Xu ${ }^{1,2}$, Bin Zhang ${ }^{1,2}$, \\ Ting Liu ${ }^{6}$, Feng Jin ${ }^{3}$ \\ ${ }^{1}$ Department of Breast Surgery, Cancer Hospital of China Medical University, Shenyang, Liaoning 110042, China; ${ }^{2}$ Department of Breast Surgery, \\ Liaoning Cancer Hospital \& Institute, Shenyang 110042, China; ${ }^{3}$ Department of Breast Surgery, the First affiliated Hospital of China Medical \\ University, Shenyang 110001, China; ${ }^{4}$ Department of Radiology, ${ }^{5}$ Department of Pathology, Liaoning Cancer Hospital \& Institute, Shenyang \\ 110042, China; ${ }^{6}$ Department of Radiology, the First affiliated Hospital of China Medical University, Shenyang 110001, China
}

Correspondence to: Feng Jin. Department of Breast Surgery, the First affiliated Hospital of China Medical University, Shenyang 110001, China. Email: jinfeng66cn@hotmail.com; Ting Liu. Department of Radiology, the First affiliated Hospital of China Medical University, Shenyang 110001, China. Email: Tingliu365@gmail.com.

Background: Patients treated with neoadjuvant chemotherapy (NAC) who achieve a pathologic complete response (pCR) can be identified preoperatively and can potentially be spared the morbidity of surgery. The objective of this retrospective study was to estimate the diagnostic accuracy of preoperative magnetic resonance imaging (MRI) in predicting pCR in patients with different molecular subtypes of breast cancer and to provide a basis for the selection of surgical methods.

Methods: We retrospectively reviewed breast MRI data from August 2015 to December 2018 of patients who underwent four or more cycles of NAC. Factors associated with radiological complete response (rCR) and pCR were analyzed in univariable and multivariable settings. The accuracy of MRI and the correlation between rCR and pCR were also analyzed in each tumor subtype.

Results: A total of 177 women with a primary tumor fulfilled the study criteria; 18 of these patients (10.2\%) achieved rCR, and 21 (11.9\%) achieved a pCR. MRI diagnosis of rCR was significantly correlated with pCR with a Spearman's correlation coefficient of 0.686 in the entire population. The sensitivity, specificity, accuracy, pCR predictive value (PPV), and non-pCR predictive value (NPV) were estimated to be 66.67\%, 97.44\%, 93.79\%, 77.78\%, and 95.60\%, respectively. Statistically significant correlations between rCR and pCR were found in Luminal B high Ki67\% ( $<<0.001)$, HER2-positive $(\mathrm{P}=0.0035)$, and triple-negative $(\mathrm{P}<0.001)$ subtypes, but not in Luminal A and Luminal B low Ki67\% subtypes. On univariate analysis, the tumor characteristics significantly associated with both rCR and pCR were small tumor, lymph node metastasis (LNM) negativity, early clinical stage, high grade, high Ki67\% index, and different molecular subtype. On multivariate logistic regression analysis, grade 3 tumors $(\mathrm{P}=0.013), \mathrm{Ki67} \% \geq 40 \%(\mathrm{P}<0.000)$, and stage I tumor $(\mathrm{P}=0.006)$ were independently associated with rCR. However, grade 3 tumors $(\mathrm{P}=0.001)$, triplenegative breast cancer (TNBC), and clinical stages I and II tumors ( $\mathrm{P}=0.003 ; \mathrm{P}=0.030)$ were independently associated with the likelihood of attaining a pCR.

Conclusions: The overall accuracy of MRI in predicting pCR in invasive breast cancer patients who received NAC was $93.8 \%$. The performance of MRI differed among molecular subtypes, and the highest PPV was found in TNBC (100\%) and Luminal B high Ki67\% (75\%) subtypes. 


\begin{abstract}
Keywords: Magnetic resonance imaging (MRI); molecular subtypes; pathologic complete response (pCR); breast cancer; neoadjuvant chemotherapy (NAC)
\end{abstract}

Submitted Sep 06, 2019. Accepted for publication Nov 12, 2019.

doi: 10.21037/qims.2019.11.16

View this article at: http://dx.doi.org/10.21037/qims.2019.11.16

\section{Introduction}

Breast cancer is still the most common cancer in the Chinese female population, with around 279,000 new patients diagnosed each year in China (1). Neoadjuvant chemotherapy (NAC) is one of the most commonly recommended treatments for local regionally advanced breast cancer, as it can improve the surgical outcomes among the patients in whom a primary surgical approach is technically not feasible. It can also avoid a poor cosmetic outcome for patients who desire breast conservation but in whom a mastectomy or a partial mastectomy is required due to an operable breast cancer (2). According to previous reports, the use of effective first-line chemotherapeutic agents and targeted therapies has yielded high rates of pathologic complete response (pCR) (3-6). However, it is still unknown whether excision of the tumor in the setting of pCR improves the risk of locoregional recurrence. Therefore, there is a keen interest in determining whether negative imaging results after systemic therapy might help identify a patient subset that could be treated safely with radiation alone without surgery. Prior studies that evaluated radiotherapy as the definitive modality for treating breast cancer patients with clinical complete response to NAC have shown high locoregional failure rates (7-9). This may be attributed to poor patient selection, as clinical examination for detecting residual disease or response is limited (10-12).

Breast magnetic resonance imaging (MRI) is a more sensitive modality to detect benign and malignant breast lesions compared with mammography and ultrasound, especially in young patients with a dense mammary gland $(13,14)$. In patients undergoing NAC, breast MRI is also an efficient noninvasive method in evaluating the efficacy of chemotherapy (15). Previous studies have suggested that a radiological complete response (rCR) has a certain correlation with pCR (16) However, there is limited research that has studied the correlation between rCR and pCR in different molecular subtypes, especially with the inclusion of the proliferation index $\mathrm{Ki} 67 \%$ in the study cohort (17). Ideally, if MRI is able to accurately distinguish
pCR from non-pCR in some specific molecular subtypes, additional mastectomy can be avoided (18). Therefore, in the current study, we sought to determine the performance of MRI after NAC in a large single-center data set to better define the accuracy of post-treatment breast MRI in the prediction of pCR. Moreover, we wanted to identify the tumor-related variables that were associated with the highest correlation between an rCR and a pCR, along with a patient population that may be most amenable to treatment without surgery based on showing an rCR.

\section{Methods}

\section{Patients and systemic treatment}

We retrospectively reviewed the breast MR imaging data from patients who underwent four or more cycles of NAC from August 2015 to December 2018, after we received approval from the Liaoning Cancer Hospital and Institute. All patients had histologically confirmed invasive breast cancer before treatment by core needle biopsy and had received NAC. Patients who underwent serial breast MRI for follow-up of the tumor response to NAC were included. Each patient received at least two MRI evaluations, one before core needle biopsy and NAC treatment, and the other at follow-up within 40 days of definitive surgery. No additional NAC treatments were performed during the period of the follow-up MRI scan and definitive surgery.

Experienced oncologists determined the regimens of chemotherapy after reviewing the clinical information of patients. Most of the patients received the anthracycline regimen with epirubicin and cyclophosphamide followed or combined with the taxane-based regimen docetaxel. HER2-positive breast cancer patients also received targeted therapy with trastuzumab. Some triple-negative breast cancer (TNBC) patients received docetaxel combined with carboplatin regimen chemotherapy. For patients who had a poor physical condition and heart disease, the docetaxel and cyclophosphamide regimen were also chosen to decrease the toxicity of chemotherapy. 


\section{Pathological classification}

All histopathology and biomarker assessments were performed by two pathologists who had at least five years' experience. The tumor specimens were fixed in formalin and stained with $\mathrm{H} \& \mathrm{E}$ to evaluate the characteristics of tumors. Tumors were classified as estrogen receptor (ER) positive or progesterone receptor $(\mathrm{PR})$ positive if immunohistochemical (IHC) staining showed stained tumor cell nuclei $\geq 1 \%$. HER2 status (positive or negative) was determined by IHC and/ or fluorescence in situ hybridization (FISH) analysis of the biopsied tissue. On IHC analysis, 3+ was considered positive, and 0 to $1+$ was considered negative. If a $2+$ result was obtained, the tissue was subsequently evaluated by FISH for HER2 gene amplification. When the HER2 to chromosome 17 centromere ratio was $\geq 2.0$, or the mean HER 2 copy number was $\geq 6$, the result was defined as positive. The proliferative index (Ki67\%) immunostaining was confined to the nucleus, five different views of the microscope were selected, and the result was obtained by averaging these five different views. We classified the Ki67\% index into three groups by values of less than $15 \%$ and more than $40 \%$. Tumors were stratified into molecular subtypes using IHC surrogate markers. The subtypes were Luminal A (ER positive, PR $\geq 20 \%$ positive, HER2 negative, Ki67\% $\leq 15 \%$ ), Luminal B (ER positive, and/or PR $<20 \%$ positive, HER2 negative or ER/PR/HER2 positive, or ER and PR positive but Ki67\% $>15 \%$ ), HER2 positive (ER and PR negative HER2 positive), and triple-negative (ER/PR/HER2 negative) groups. We also stratified Luminal B into two different groups according to the Ki67\% index, which was defined as the Luminal B low Ki67\% group by Ki67\% < 40\% and the Luminal B high Ki67\% group by Ki67\% $\geq 40 \%$. After surgery, we used the MillerPayne grading system to evaluate the response to NAC (6). Grades 1 and 2 were categorized as stable. Grades 3 and 4 were categorized as a partial pathological response (pPR), and grade 5 was categorized as pCR. Residual ductal carcinoma in situ was excluded from pCR regardless of whether lymph node $(\mathrm{LN})$ metastasis was present.

\section{Breast MRI and interpretation}

Patients were scanned with the 1.5-Tesla MRI system (Sigma Excite HD; GE Healthcare, USA) with a resolute 8-channel high-definition breast coil. The patient was placed in the prone position on the examination bed, with bilateral breasts naturally hanging in two circles of the breast coil during the examination. First, sagittal fat-saturated T2-weighted fast-spin echo images of the bilateral breasts were recorded using the following parameters: repetition time/echo time, 4,040 ms/81 ms; echo train length, 19; slice thickness, $5 \mathrm{~mm}$; slice gap, $1 \mathrm{~mm}$; field of view, $220 \mathrm{~mm}$; matrix size, $320 \times 224$; number of excitations, 2; imaging time, 1 min $41 \mathrm{~s}$. Then, unenhanced and enhanced sequences of axial fat-saturated gradient recalled echo T1-weighted images were acquired with the following parameters: repetition time/echo time/inversion time, $6.1 \mathrm{~ms} / 2.9 \mathrm{~ms} / 13 \mathrm{~ms}$; flip angle, $10^{\circ}$; slice thickness, $3.2 \mathrm{~mm}$; slice gap, $0 \mathrm{~mm}$; field of view, $360 \mathrm{~mm}$; matrix size, $350 \times 350$; number of excitations, 0.8 ; imaging time, $58 \mathrm{~s}$ per phase; 8 phases of enhancement. Gadopentetate dimeglumine was administered at a dose of $15 \mathrm{~mL}$ and a rate of $2.5 \mathrm{~mL} / \mathrm{s}$, followed by a $20-\mathrm{mL}$ saline flush given with an automatic injector.

Two radiologists with at least 5 years' experience in interpreting breast MRI, performed the MRI tumor size measurement using the same measurement standard. The two radiologists independently reviewed the MRI image, and they were blinded to the pathology results. The baseline size of a lesion was defined as the maximal diameter in a single dimension measured by pretreatment MRI, the residual tumor size on the final MRI was measured by subtracting the pre-contrast images from the post-contrast images, and the maximum intensity projections were generated from the subtraction images. MRI measured the tumor size in three dimensions for each case. When there was no discernible enhancement or faint enhancement equal to the background of normal breast tissue at the earlier lesion site, the case was determined as showing an rCR on MRI.

\section{Statistical analysis}

SPSS Statistics (version 22.0) was used for all analyses. Continuous variables such as age, physical examination tumor size, baseline and final MRI tumor size, and final pathologic tumor size, and categorical variables, including tumor grade, and molecular subtype, were separately calculated. Continuous variables were expressed as mean and standard deviation, and categorical variables were expressed as absolute values and percentages which were used by the Chi-square test or Fisher's exact test respectively, to determine the statistical significance. Pearson and Spearman's correlation coefficients between the tumor size on MRI and pathological tumor size were estimated. Basic statistical indicators, such as accuracy, sensitivity, and specificity, used to assess the accuracy of MRI in detecting rCR after NAC, were calculated. The efficacy of MRI was 
Table 1 Clinical and tumor characteristics

\begin{tabular}{lc}
\hline Characteristics & Number, $\mathrm{n}(\%)$ \\
\hline Age (y), mean \pm SD & $50.8 \pm 9.2$ \\
Menopause & \\
Yes & $87(49.2)$ \\
No & $90(50.8)$ \\
Regimens of chemotherapy & \\
Inclusion of anthracyclines & $143(80.8)$ \\
Non-inclusion of anthracyclines & $34(19.2)$ \\
Surgery & \\
Modified radical mastectomy & $169(95.5)$ \\
Breast-conserving surgery & $2(1.1)$ \\
Others & $6(3.4)$ \\
Pathological classification & \\
Invasive ductal carcinoma & $135(76.3)$ \\
Mix & $29(16.4)$ \\
Other & $13(7.3)$ \\
Miller classification & $38(21.5)$ \\
G1 & $72(40.7)$ \\
G2 & $43(24.3)$ \\
G3 & $21(11.9)$ \\
G4 & \\
\hline
\end{tabular}

measured by predictive values, including PPV and NPV. Multivariable logistic regression was used to examine the simultaneous effects of multiple factors. $\mathrm{P}<0.05$ was considered to indicate statistical significance.

\section{Results}

\section{Patients' characteristics}

A total of 223 patients with invasive breast cancer who received NAC treatment at Liaoning Cancer Hospital \& Institute from 2015 to 2018 were included. Among them, 177 patients with an evaluable primary endpoint, including MRI and final pathology, were studied. The mean age of the study cohort was 51 years (range, 25-68 years) at baseline. A summary of patient and tumor characteristics is shown in Table 1.

\section{Pathological classification}

Most tumors were diagnosed as stage II (54.2\%) or stage III
(42.9\%). Only 5 (2.8\%) patients were diagnosed with stage I. Hormone receptor, and HER2 were positive in $65.5 \%$ and $38.4 \%$ of patients, respectively. Luminal A, Luminal B low Ki67\% (<40\%), Luminal B high Ki67\% ( $\geq 40 \%)$, HER2 positive, and triple-negative were found in $6.2 \%, 27.1 \%$, $33.9 \%, 17.5 \%$, and $15.3 \%$ of patients, respectively.

\section{Systemic treatment and primary endpoints}

The anthracycline regimen with epirubicin and cyclophosphamide and a taxane was completed in 143 $(80.8 \%)$ patients with an average of at least four cycles, and the other regimens included taxane and carboplatin (15.8\%), and taxane and cyclophosphamide (3.4\%). We used different chemotherapy regimens for the patients who had different molecular subgroups in terms for individualized treatments $(\mathrm{P}=0.002)$. However, there was no significant difference between the $\mathrm{pCR}$ group and the non-pCR group $(\mathrm{P}=0.617)$. In addition, among the $68 \mathrm{HER} 2$-positive breast tumors, $33(48.5 \%)$ received trastuzumab. After NAC, all patients underwent surgery. The total clinical response rate was $75.2 \%, 22.1 \%$ of patients achieved stable disease, $65.0 \%$ of patients achieved a PR, and the remaining $10.2 \%$ of patients achieved a CR. The total pathological response rate was $76.9 \%$, which included $\geq \mathrm{G} 3$ pathological response.

\section{Performance of breast MRI}

The primary tumor size assessed by MRI before treatment was $4.03 \pm 1.86 \mathrm{~cm}$ (range, $1.3-11.6 \mathrm{~cm}$ ). After completion of NAC, the residual tumor size measured by MRI was $2.29 \pm 1.41 \mathrm{~cm}$ (range, $0-6.3 \mathrm{~cm}$ ). The final residual tumor size measured by pathology was $2.02 \pm 1.42 \mathrm{~cm}$ (range, $0-8 \mathrm{~cm}$ ).

Of the 177 patients, a pCR was achieved in $21(11.9 \%)$ patients, and 14 (66.7\%) patients were correctly diagnosed by MRI (Figure 1). Of the 159 patients who showed non-rCR, 152 (95.6\%) also showed non-pCR, but the remaining 7 (4.4\%) showed a pCR (Figure 2). Thus, rCR was significantly correlated with pCR in the whole cohort $(\mathrm{P}<0.001$, Table 2). Sensitivity, specificity, accuracy (ACC), pCR predictive value (PPV), and non-pCR predictive value (NPV) were estimated to be $66.67 \%, 97.44 \%, 93.79 \%$, $77.78 \%$, and $95.60 \%$, respectively (Table 2).

We also performed a comparison between pCR and rCR in each subtype group. Statistically significant correlations between $\mathrm{rCR}$ and $\mathrm{pCR}$ were found in Luminal $\mathrm{B}$ high Ki67\% $(\mathrm{P}<0.001)$, HER2-positive $(\mathrm{P}=0.0035)$, and triplenegative $(\mathrm{P}<0.001)$ subtypes, but not in Luminal $\mathrm{A}$, and 

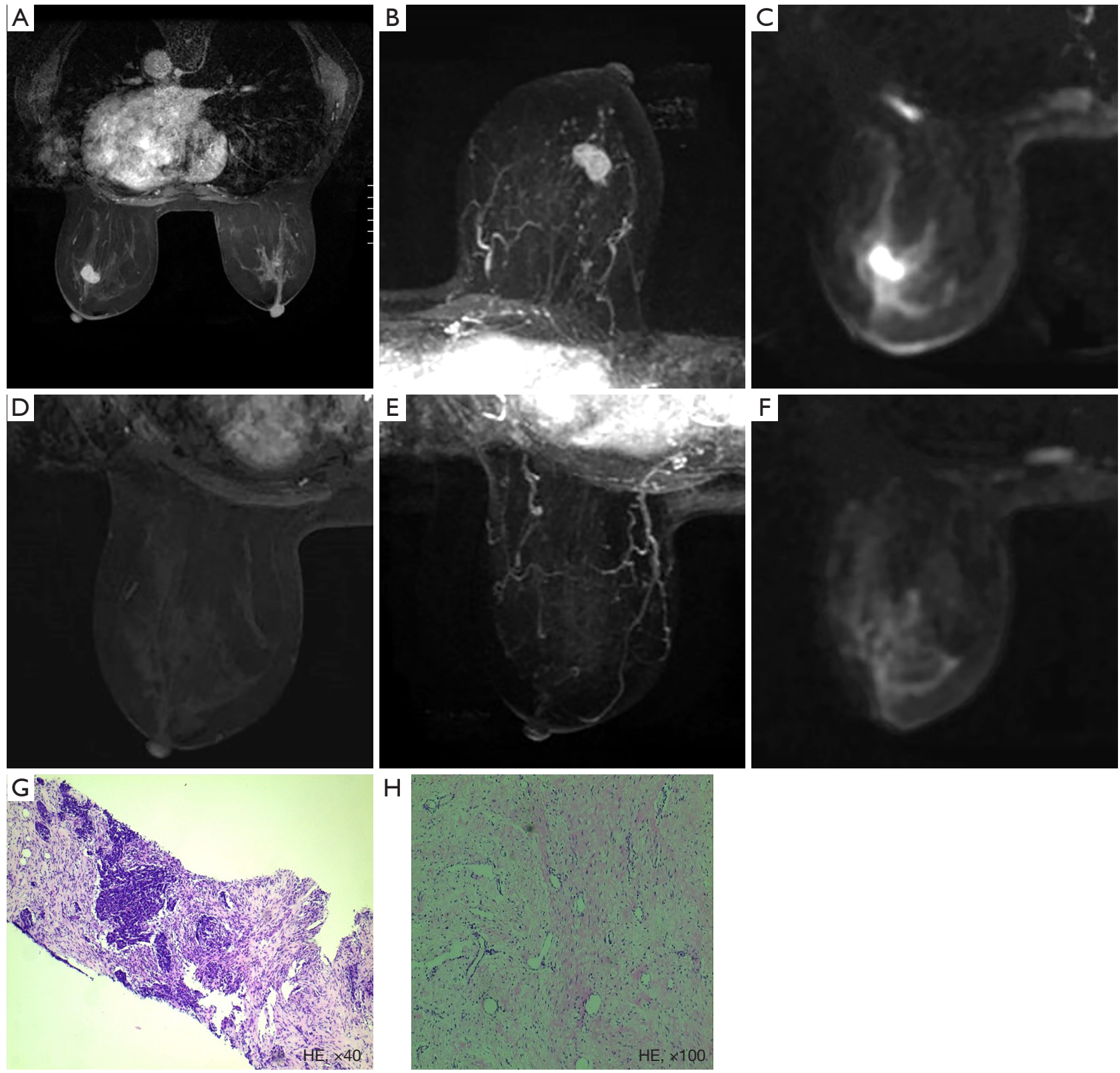

Figure 1 Images of a 68-year-old woman with a diagnosis of Luminal B High Ki67\% breast cancer before and after treatment. MR images and pathological findings of a case with a rCR and pCR. Baseline MRI scan before (A, DCE image; B, MIP; and C, the DWI sequence) and after completion of NAC treatment (D, DCE image; E, MIP; and F, the DWI sequence). No enhancement was detected by MRI after NAC. Core needle biopsy confirmed invasive ductal carcinoma (G). After 6 cycles of NAC, histopathologic examination confirmed a pCR (H). rCR, radiographic complete response; pCR, pathologic complete response; MRI, magnetic resonance imaging; NAC, neoadjuvant chemotherapy; DCE, dynamic contrast-enhanced; MIP, maximum intensity projection; DWI, diffusion-weighted imaging.

Luminal B low Ki67\% subtypes. The sensitivity, specificity, ACC, PPV, and NPV for correlations between rCR and pCR for each subtype are shown in Table 2.

For the Luminal A subtype, the NPV was $100 \%$, with a perfect concordance in all 10 patients with non-rCR, which was pathologically diagnosed as non-pCR. In contrast to the Luminal subtype, the PPV and specificity for the triplenegative subtype were both $100 \%$. The sensitivity was 

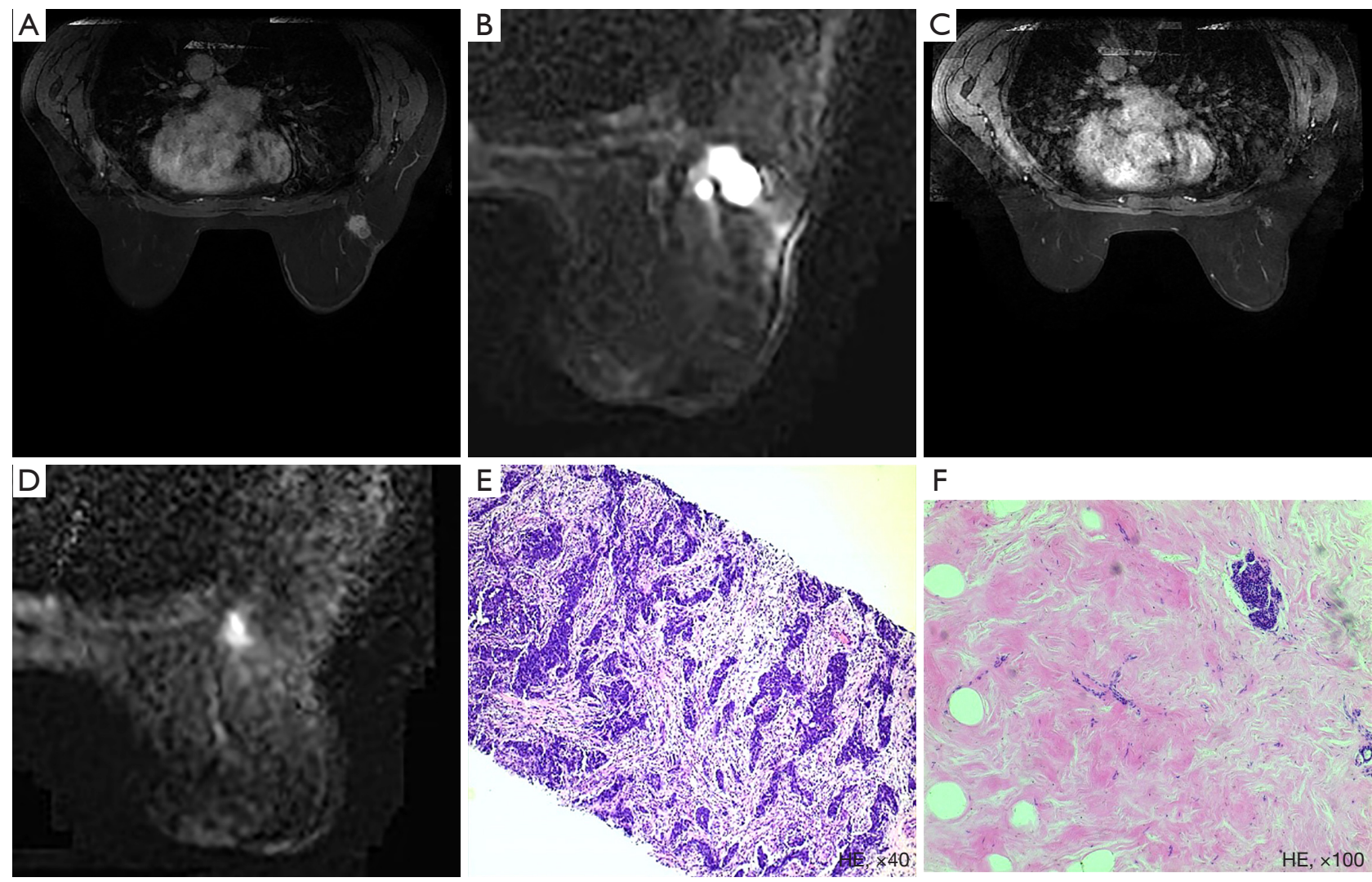

Figure 2 Images of a 65-year-old woman with a diagnosis of HER2-positive breast cancer before and after treatment. Core needle biopsy confirmed invasive ductal carcinoma (E). After 6 cycles of NAC, histopathologic examination confirmed a pCR. Note residual fibrosis, inflammatory changes, and the complete absence of malignant cells (F). Baseline (A,B,C,D) MR images of a HER2-positive breast cancer before (A, DCE image; B, DWI sequence) and after (C, DCE image; D, DWI sequence) NAC. After NAC, no mass was detected but scattered dendritic enhancement was noted, which predicted non-rCR. pCR, pathologic complete response; NAC, neoadjuvant chemotherapy; DCE, dynamic contrast-enhanced; DWI, diffusion-weighted imaging.

Table 2 Comparison of breast MRI performance among tumor subtypes after NAC

\begin{tabular}{|c|c|c|c|c|c|c|}
\hline Molecular subtype & Accuracy & Sensitivity & Specificity & PPV & NPV & $P$ value \\
\hline Luminal A & $90.91(10 / 11)$ & $0(0 / 0)$ & $90.91(10 / 11)$ & $0(0 / 11)$ & $100(10 / 10)$ & NS \\
\hline Luminal B low Ki67\% & $97.92(47 / 48)$ & $0(0 / 1)$ & $100(47 / 47)$ & $0(0 / 0)$ & $97.92(47 / 48)$ & NS \\
\hline Luminal B high Ki67\% & $93.33(56 / 60)$ & $75.00(6 / 8)$ & $96.15(50 / 52)$ & $75.00(6 / 8)$ & $96.15(50 / 52)$ & $<0.000$ \\
\hline Triple negative & $92.59(25 / 27)$ & $75.00(6 / 8)$ & $100(19 / 19)$ & $100(6 / 6)$ & $90.48(19 / 21)$ & $<0.000$ \\
\hline
\end{tabular}

The data are shown as \% (No. of patients/total No.). HER2, human epidermal growth factor receptor; NPV, negative predictive value; NS, nonsignificant; PPV, positive predictive value. P values were calculated by comparing the 5 molecular subtypes for each performance measure (sensitivity, specificity, NPV, PPV, and accuracy) with magnetic resonance breast imaging using the Chi-square test. 

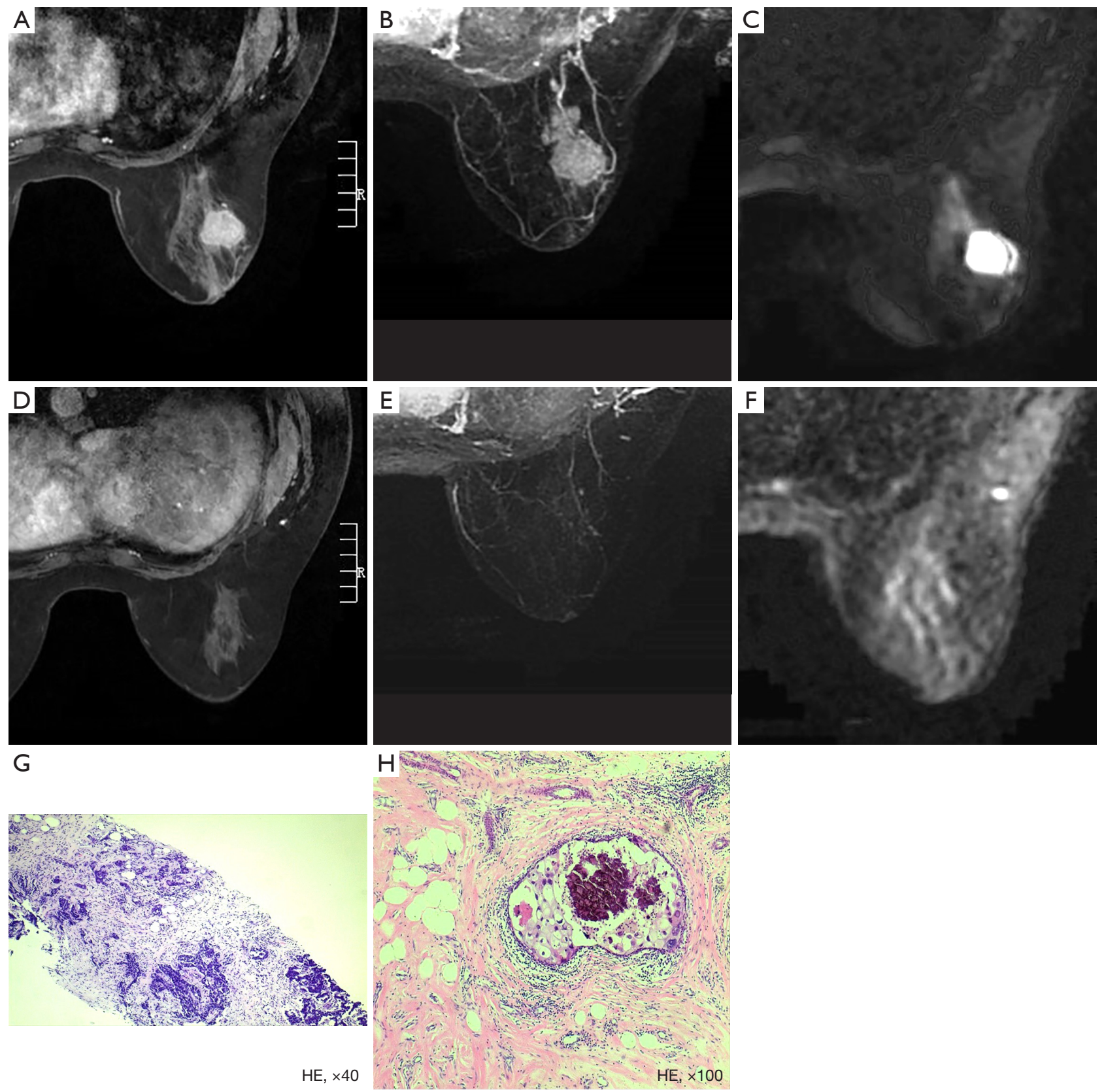

Figure 3 Images of a 68-year-old woman with a diagnosis of HER2-positive breast cancer before and after treatment. MR images of HER2positive breast cancer before (A, DCE image; B, MIP; and C, DWI sequence) and after (D, DCE image; E, MIP; and F, DWI sequence) NAC; the tumor was no longer enhanced, yielding a rCR. Pre-NAC pathologically confirmed invasive ductal carcinoma (G). Post-NAC pathological examination showed residual disease with DCIS and some carcinoma cells invading out of the duct (H). rCR, radiographic complete response; NAC, neoadjuvant chemotherapy; DCE, dynamic contrast-enhanced; MIP, maximum intensity projection; DWI, diffusion-weighted imaging.

$75.0 \%$, which was the highest score among all the subtypes, suggesting that MRI is a valuable modality for predicting pCR in this subtype. For the HER2 enriched subtype, sensitivity (50.0\%), specificity (96.3\%), ACC (90.32\%), PPV (66.67\%), and NPV (92.86\%) were relatively low (Figure 3). In contrast, sensitivity (75.0\%), specificity 
(96.15\%), ACC (93.33\%), PPV (75.0\%), and NPV (96.15\%) were relatively high for the Luminal B high Ki67\% subtype.

\section{Univariate and multivariate regression analyses of covariates affecting MRI accuracy}

Among all 177 patients, 18 (10.2\%) achieved an rCR, and $21(11.9 \%)$ achieved a pCR. Tumor characteristics (lower tumor diameters, LN metastasis negativity on MRI, low clinical stage of the tumor, high grade, high Ki67\% index, and different molecular subtypes) were significantly associated with both rCR and pCR (Table 3).

On univariate analysis, hormone receptor (HR) negativity was associated with pCR but not with rCR. MRI diagnosis of rCR was significantly correlated with pCR with a Spearman's correlation coefficient of 0.686 for the entire population $(\mathrm{P}<0.001)$. Variables that were associated with $\mathrm{rCR}$ included primary tumor diameters measured by hand and MRI $(\mathrm{P}=0.008, \mathrm{P}=0.005)$, tumor diameter after NAC measured by MRI $(\mathrm{P}<0.001)$, clinical stage $(\mathrm{P}=0.027), \mathrm{Ki} 67 \%$ index $(\mathrm{P}=0.005)$, tumor grade $(\mathrm{P}=0.020)$, and molecular subtype $(\mathrm{P}=0.010)$. In addition to the above factors, variables that were associated with pCR included primary LNM by MRI $(\mathrm{P}=0.003)$ and HR status $(\mathrm{P}=0.020)$ (Table 4).

Multivariate logistic regression analysis of variables that significantly influenced rCR and pCR was performed with a final model that included clinical stage, Ki67\% index, tumor grade, and molecular subtype. Variables that were independently associated with rCR included grade 3 tumor $(\mathrm{P}=0.013), \mathrm{Ki} 67 \% \geq 40 \%(\mathrm{P}<0.000)$, and stage $\mathrm{I}$ tumor $(\mathrm{P}=0.006)$. Variables that were independently associated with the likelihood of attaining a pCR were grade 3 tumors $(\mathrm{P}=0.001)$, TNBC and clinical stages I and II tumors $(\mathrm{P}=0.003 ; \mathrm{P}=0.030)($ Table 5$)$.

\section{Discussion}

NAC plays an especially key role in routine breast cancer treatment. A pCR after NAC has been accepted practice since the earliest neoadjuvant trials. A meta-analysis clearly demonstrated that pCR resulted in improved survival, irrespective of the treatment received, and it is a critically important surrogate endpoint for better prognosis (19). However, the definition of pCR is still controversial, and there appears to be substantial heterogeneity in the definition across different trials, leading to difficulty in comparing the outcomes. We used G5 as a standard for pCR, which indicated that DCIS or malignant cells are not visualized in sections from the site of the tumor. Thus, we obtained a low pCR rate of about $11.9 \%$, compared to the other research (20-22). One of the reasons for this occurrence is that we used a more stringent pCR standard, and the other reason for this occurrence is that only a few patients were subjected to the anti-HER2 target therapy, even though, in those who used the targeted therapy, a low pCR rate $(12.9 \%)$ was achieved. However, the rCR results were found to significantly correlate with pCR. These results are consistent with the previously reported data $(23,24)$. In our study, we wanted not only to ensure that rCR was significantly correlated with pCR but also identify the factors that can maximally influence the $\mathrm{rCR}$ and pCR rates. We obtained a conclusion from Table 4 that tumor diameter measured by MRI after NAC was the most important factor correlated with rCR and pCR. Although the other factors, such as primary tumor diameter measured by hand and MRI, clinical-stage, Ki67\% index, tumor grade, and molecular subtype, were significantly correlated with $\mathrm{rCR}$ and $\mathrm{pCR}$, the statistical significance was relatively low. This may be because of the small tumor size and early tumor clinical classification, and a stronger ability for proliferation being able to display a high probability of response and a higher chance to achieve an $\mathrm{rCR}$ and $\mathrm{pCR}$ after NAC $(25,26)$. Primary LNM by MRI and hormone receptor can also influence the $\mathrm{pCR}$, and patients with nonmetastatic LNs and HR negativity can easily achieve a pCR. These results are consistent with the univariate analysis, which can cause rCR and pCR.

We also investigated the accuracy of MRI in predicting pCR among breast cancer patients after NAC, especially with a focus on its value with respect to each tumor subtype. In 2013, the St. Gallen Expert Consensus recommended the classification based on IHC markers (27). We made a slight change, and we divided Luminal B breast cancer into two different groups, high Ki67\%, and low Ki67\% groups. We found out that the accuracy of rCR was significantly correlated with pCR, especially in Luminal B high Ki67\%, HER2-positive, and triple-negative subtypes. However, there are still some differences between these three groups.

On the one hand, Luminal B high Ki67\% and HER2 positive subtypes had a high NPV, but the PPV was low. This suggests that remnant lesions found by MRI are reliable markers of non-pCR for these two subtypes. However, rCR diagnosed by MRI in these subtypes may have been overestimated.

On the other hand, MRI accurately predicted pCR in the triple-negative subtype with a high degree of sensitivity, specificity, accuracy, PPV, and NPV, compared to the 
Table 3 Patient characteristics in breast radiographic and pathologic complete response

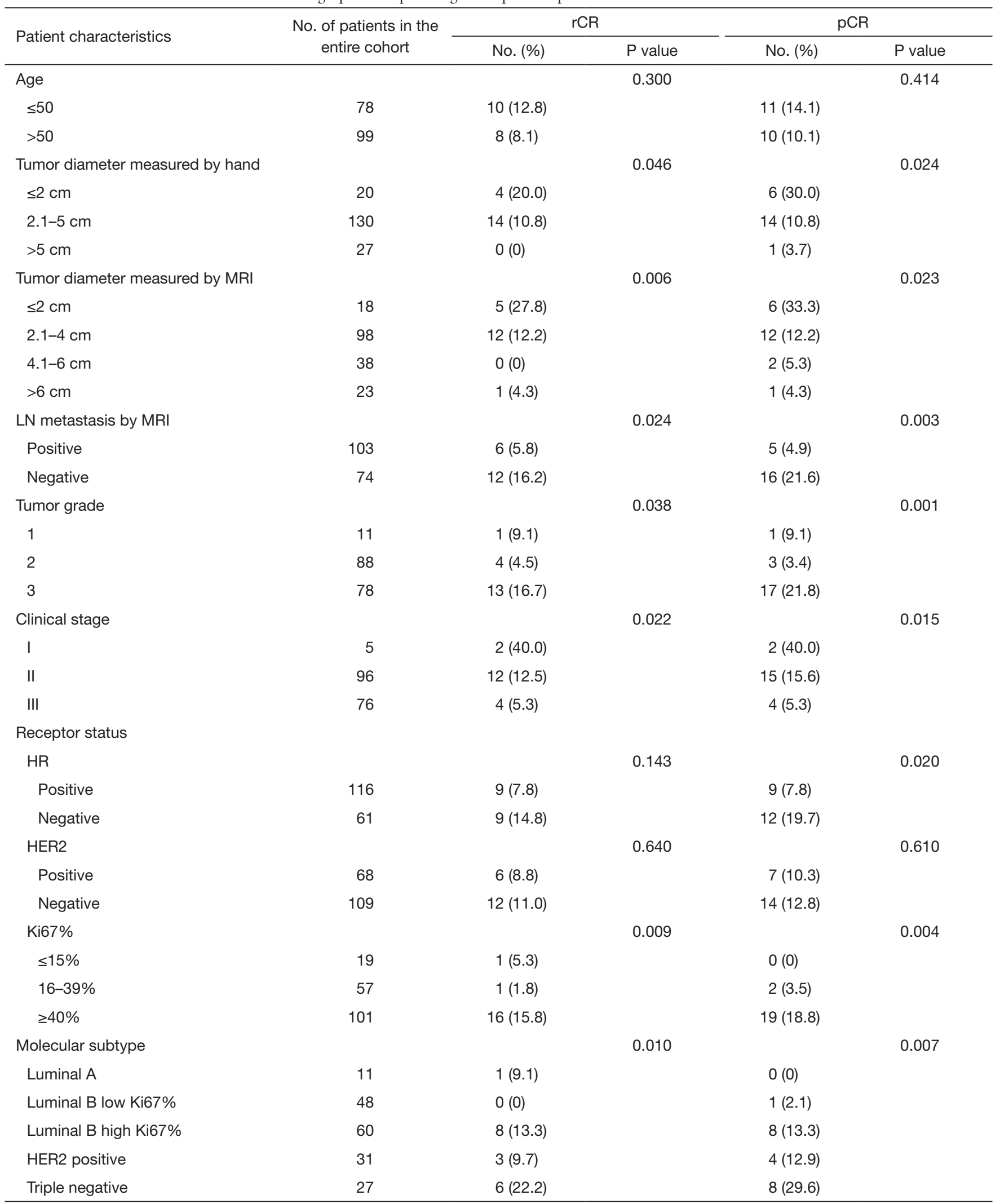


Table 4 The correlation coefficient according to the clinical and tumor characteristics

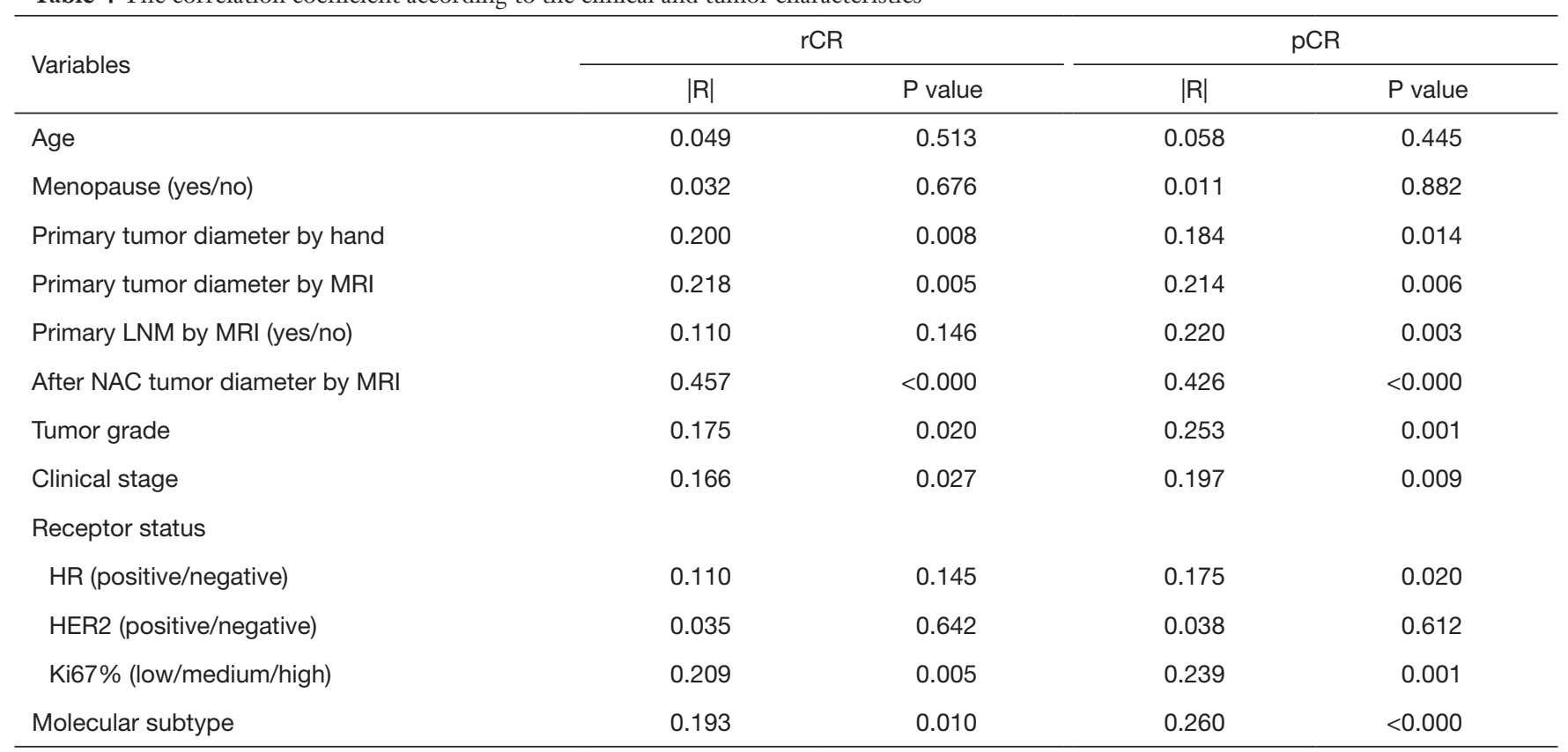

MRI, magnetic resonance imaging; NAC, neoadjuvant chemotherapy.

Table 5 Multivariate regression analysis according to the clinical and tumor characteristics

\begin{tabular}{|c|c|c|c|c|}
\hline Variables & \multicolumn{2}{|c|}{ rCR } & \multicolumn{2}{|c|}{$\mathrm{pCR}$} \\
\hline \multicolumn{5}{|l|}{ Tumor grade } \\
\hline 1 & -0.94 & 0.419 & -1.25 & 0.303 \\
\hline 2 & -1.73 & 0.013 & -2.71 & 0.001 \\
\hline \multicolumn{5}{|l|}{ Clinical stage } \\
\hline 1 & 3.60 & 0.006 & 4.27 & 0.003 \\
\hline II & 1.06 & 0.103 & 1.446 & 0.030 \\
\hline III & 0 & - & 0 & - \\
\hline$\geq 40 \%$ & 0 & - & 0 & - \\
\hline \multicolumn{5}{|l|}{ Molecular subtype } \\
\hline Luminal A & 17.76 & - & -0.316 & - \\
\hline Luminal B low Ki67\% & -18.23 & 0.997 & -0.98 & 0.524 \\
\hline Luminal B high Ki67\% & -0.945 & 0.162 & -1.61 & 0.022 \\
\hline HER2 positive & -1.38 & 0.114 & -1.71 & 0.045 \\
\hline Triple negative & 0 & - & 0 & - \\
\hline
\end{tabular}

rCR, radiological complete response; pCR, pathologic complete response. 
other subtypes. These results are consistent with earlier reports (23,28-30). In our cohort, Luminal A and Luminal B low Ki67\% breast cancers were less likely to show an rCR or pCR to neoadjuvant therapy, as no Luminal A patients achieved a pCR, and no Luminal B low Ki67\% patients achieved an rCR; even though they obtained a high specificity, ACC, and NPV, they obtained a $0 \%$ for sensitivity and PPV. These results are consistent with those in the study by Dave (31). A larger cohort is needed before drawing any definitive conclusions, especially for the Luminal A and Luminal B low Ki67\% subtypes. However, MRI still obtained a good NPV for these two molecular subgroups. This means that if the MRI shows a residual tumor, the accuracy is still high. Some studies $(32,33)$ have shown that $\mathrm{rCR}$ is not accurate enough to serve as a surrogate marker for pCR on MRI after NAC. The major difference between our study and those studies was that we used the modified molecular classification methods, which is consistent with the clinical consensus. In addition, we used a stricter diagnosis standard for rCR patients. An rCR was defined as the absence of any enhancement in the tumor zone on both DCE-MRI and DWI-MRI images. This might have caused a major difference between our results and the results of those studies. However, those studies also found that ER-negative or HER2-positive breast cancer was more accurate than ER-positive breast cancer, which is consistent with our result.

Furthermore, although pCR is still the gold standard for NAC, rCR can inform the clinician that this type of patient may achieve a good prognosis (34). In our study, different clinical and tumor characteristics influenced the patients' chances of achieving rCR and pCR. Smaller tumor burden and stronger tumor proliferative ability made it easier to achieve pCR after NAC and diagnosis by MRI. It is easier to achieve pCR in patients with negative hormone receptors, but it is not very helpful in the diagnosis of rCR after NAC. NAC might also induce reactive changes, such as fibrosis and inflammation, and may result in nonspecific contrast enhancement leading to an underestimated or overestimated tumor size by MRI (35). In addition, the ability of different molecular subtypes of breast cancer to achieve a pCR is also different. The correlation between pathologic response and molecular subtypes in patients with breast cancer was strongest in the triple-negative subtype, lower in the Luminal B high Ki67\% and HER2-positive subtypes, and lowest in the Luminal B low Ki67\% and Luminal A subtypes. This result was similar to that found in the study by Bufi et al., in which an adequate performance in predicting a pCR in the triple-negative subtype and a lower performance in the Luminal A subtype were found (36). $\mathrm{Ki} 67 \%$ is another important finding of this study. When $\mathrm{Ki} 67 \%$ was more than $40 \%$, the pCR rate after NAC increased significantly, and the diagnostic rate of rCR also increased significantly. When Ki67\% $\geq 40 \%$ was introduced in Luminal B typing, it was found that pCR was more easily achieved in the high Ki67\% Luminal B subtype, and its pCR rate was almost the same as that in HER2-positive breast cancer. From the multivariate analysis, we also observed that grade 3 compared with grade 2, stages I and II compared with stage III, and the triple-negative subtype compared with the Luminal B high Ki67\% and HER2positive subtypes more easily achieved pCR. Although the difference in the molecular subtype was not significant in the diagnosis of rCR, high Ki67\% was an important predictor of rCR compared with low Ki67\%.

There are several limitations to our study. The number of cases in our study was small, especially the number of patients with pCR and rCR. However, since this was a single-center study, we could ensure consistent study procedures (treatment regimen and imaging follow-up), and standardized data analysis methods were used for imaging and pathological evaluation. Despite the small number of subjects, we could thoroughly investigate the predictive role of all considered variables, and our results were consistent with those in the literature. In addition, our patients had $17.5 \%$ HER 2 positivity rates, but only $45.6 \%$ of them used trastuzumab target therapy, and this would decrease the pCR rates in the HER2 positive subtype. Finally, the specificities of the MR contrast type and infusion protocol were not determined in this retrospective study.

In conclusion, with a strict standard for diagnosing rCR and $\mathrm{pCR}$, the accuracy of breast MRI was high for predicting a pCR in patients who received NAC for invasive breast cancer. Sensitivity, NPV, PPV, and accuracy of MRI in predicting a pCR differed significantly among breast cancer molecular subtypes, and the highest PPV was found in TNBC (100\%) and Luminal B high Ki67\% (75\%) subtypes.

\section{Acknowledgments}

None.

\section{Footnote}

Conflicts of Interest: The authors have no conflicts of interest to declare. 
Ethical Statement: This study received approval from the Liaoning Cancer Hospital and Institute.

\section{References}

1. Bray F, Ferlay J, Soerjomataram I, Siegel RL, Torre LA, Jemal A. Global cancer statistics 2018: GLOBOCAN estimates of incidence and mortality worldwide for 36 cancers in 185 countries. CA Cancer J Clin 2018;68:394-424.

2. Adnan Aydiner AI, Atilla Soran. Breast Cancer A Guide to Clinical Practice. part IV page 244 ISBN:978-3-31996946-6, 2019.

3. Baselga J, Bradbury I, Eidtmann H, Di Cosimo S, de Azambuja E, Aura C, Gómez H, Dinh P, Fauria K, Van Dooren V, Aktan G, Goldhirsch A, Chang TW, Horváth Z, Coccia-Portugal M, Domont J, Tseng LM, Kunz G, Sohn JH, Semiglazov V, Lerzo G, Palacova M, Probachai V, Pusztai L, Untch M, Gelber RD, Piccart-Gebhart M; NeoALTTO Study Team. Lapatinib with trastuzumab for HER2-positive early breast cancer (NeoALTTO): a randomised, open-label, multicentre, phase 3 trial. Lancet 2012;379:633-40.

4. Buzdar AU, Ibrahim NK, Francis D, Booser DJ, Thomas ES, Theriault RL, Pusztai L, Green MC, Arun BK, Giordano SH, Cristofanilli M, Frye DK, Smith TL, Hunt KK, Singletary SE, Sahin AA, Ewer MS, Buchholz TA, Berry D, Hortobagyi GN. Significantly higher pathologic complete remission rate after neoadjuvant therapy with trastuzumab, paclitaxel, and epirubicin chemotherapy: results of a randomized trial in human epidermal growth factor receptor 2-positive operable breast cancer. J Clin Oncol 2005;23:3676-85.

5. Gianni L, Eiermann W, Semiglazov V, Manikhas A, Lluch A, Tjulandin S, Zambetti M, Vazquez F, Byakhow M, Lichinitser M, Climent MA, Ciruelos E, Ojeda B, Mansutti M, Bozhok A, Baronio R, Feyereislova A, Barton C, Valagussa P, Baselga J. Neoadjuvant chemotherapy with trastuzumab followed by adjuvant trastuzumab versus neoadjuvant chemotherapy alone, in patients with HER2positive locally advanced breast cancer (the NOAH trial): a randomised controlled superiority trial with a parallel HER2-negative cohort. Lancet 2010;375:377-84.

6. Semiglazov V, Eiermann W, Zambetti M, Manikhas A, Bozhok A, Lluch A, Tjulandin S, Sabadell MD, Caballero A, Valagussa P, Baselga J, Gianni L. Surgery following neoadjuvant therapy in patients with HER2 positive locally advanced or inflammatory breast cancer participating in the NeOAdjuvant Herceptin (NOAH) study. Eur J Surg
Oncol 2011;37:856-63.

7. Baillet F, Rozec C, Ucla L, Chauveinc L, Housset M, Weil $M$. Treatment of locally advanced breast cancer without mastectomy.5- and 10-year results of 135 tumors larger than 5 centimeters treated by external beam therapy, brachytherapy, and neoadjuvant chemotherapy. Ann N Y Acad Sci 1993;698:264-70.

8. Mauriac L, MacGrogan G, Avril A, Durand M, Floquet A, Debled M, Dilhuydy JM, Bonichon F. Neoadjuvant chemotherapy for operable breast carcinoma larger than $3 \mathrm{~cm}$ : a unicentre randomized trial with a 124-month median follow-up. Institut Bergonie Bordeaux Groupe Sein (IBBGS). Ann Oncol 1999;10:47-52.

9. Ring A, Webb A, Ashley S, Allum WH, Ebbs S, Gui G, Sacks NP, Walsh G, Smith IE. Is surgery necessary after complete clinical remission following neoadjuvant chemotherapy for early breast cancer? J Clin Oncol 2003;21:4540-5.

10. Chagpar AB, Middleton LP, Sahin AA, Dempsey P, Buzdar AU, Mirza AN, Ames FC, Babiera GV, Feig BW, Hunt KK, Kuerer HM, Meric-Bernstam F, Ross MI, Singletary SE. Accuracy of physical examination, ultrasonography, and mammography in predicting residual pathologic tumor size in patients treated with neoadjuvant chemotherapy. Ann Surg 2006;243:257-64.

11. Kuhl C, Weigel S, Schrading S, Arand B, Bieling H, König R, Tombach B, Leutner C, Rieber-Brambs A, Nordhoff D, Heindel W, Reiser M, Schild HH. Prospective multicenter cohort study to refine management recommendations for women at elevated familial risk of breast cancer: the EVA Trial. J Clin Oncol 2010;28:1450-7.

12. Segara D, Krop IE, Garber JE, Winer E, Harris L, Bellon JR, Birdwell R, Lester S, Lipsitz S, Iglehart JD, Golshan M. Does MRI predict pathologic tumor response in women with breast cancer undergoing preoperative chemotherapy? J Surg Oncol 2007;96:474-80.

13. Suvannarerg V, Chitchumnong P, Apiwat W, Lertdamrongdej L, Tretipwanit N, Pisarnturakit P, Sitthinamsuwan P, Thiravit S, Muangsomboon K, Korpraphong P. Diagnostic performance of qualitative and quantitative shear wave elastography in differentiating malignant from benign breast masses, and association with the histological prognostic factors. Quant Imaging Med Surg 2019;9:386-98

14. Eghtedari M, Ma J, Fox P, Guvenc I, Yang WT, Dogan BE. Effects of magnetic field strength and $b$ value on the sensitivity and specificity of quantitative breast diffusionweighted MRI. Quant Imaging Med Surg 2016;6:374-80. 
15. Gu YL, Pan SM, Ren J, Yang ZX, Jiang GQ. Role of Magnetic Resonance Imaging in Detection of Pathologic Complete Remission in Breast Cancer Patients Treated With Neoadjuvant Chemotherapy: A Meta-analysis. Clin Breast Cancer 2017;17:245-55.

16. Williams M, Eatrides J, Kim J, Talwar H, Esposito N, Szabunio M, Ismail-Khan R, Kiluk J, Lee M, Laronga C, Khakpour N. Comparison of breast magnetic resonance imaging clinical tumor size with pathologic tumor size in patients status post-neoadjuvant chemotherapy. Am J Surg 2013;206:567-73.

17. Loo CE, Rigter LS, Pengel KE, Wesseling J, Rodenhuis S, Peeters MJ, Sikorska K, Gilhuijs KG. Survival is associated with complete response on MRI after neoadjuvant chemotherapy in ER-positive HER2 negative breast cancer. Breast Cancer Res 2016;18:82.

18. Ogston KN, Miller ID, Payne S, Hutcheon AW, Sarkar TK, Smith I, Schofield A, Heys SD. A new histological grading system to assess response of breast cancers to primary chemotherapy: prognostic significance and survival. Breast 2003;12:320-7.

19. Cortazar P, Zhang L, Untch M, Mehta K, Costantino JP, Wolmark N, Bonnefoi H, Cameron D, Gianni L, Valagussa P, Swain SM, Prowell T, Loibl S, Wickerham DL, Bogaerts J, Baselga J, Perou C, Blumenthal G, Blohmer J, Mamounas EP, Bergh J, Semiglazov V, Justice R, Eidtmann H, Paik S, Piccart M, Sridhara R, Fasching PA, Slaets L, Tang S, Gerber B, Geyer CE, Pazdur R, Ditsch N, Rastogi P, Eiermann W, von Minckwitz G. Pathological complete response and long-term clinical benefit in breast cancer: the CTNeoBC pooled analysis. Lancet 2014;384:164-72.

20. De Los Santos JF, Cantor A, Amos KD, Forero A, Golshan M, Horton JK, Hudis CA, Hylton NM, McGuire K, Meric-Bernstam F, Meszoely IM, Nanda R, Hwang ES. Magnetic resonance imaging as a predictor of pathologic response in patients treated with neoadjuvant systemic treatment for operable breast cancer. Translational Breast Cancer Research Consortium trial 017. Cancer 2013;119:1776-83.

21. Bouzón A, Acea B, Soler R, Iglesias A, Santiago P, Mosquera J, Calvo L, Seoane-Pillado T, Garcia A. Diagnostic accuracy of MRI to evaluate tumour response and residual tumour size after neoadjuvant chemotherapy in breast cancer patients. Radiol Oncol 2016;50:73-9.

22. Diguisto C, Ouldamer L, Arbion F, Vilde A, Body G. MRI evaluation of residual breast cancer after neoadjuvant chemotherapy: influence of patient, tumor and chemotherapy characteristics on the correlation with pathological response. Anticancer Res 2015;35:581-5.

23. Fukuda T, Horii R, Gomi N, Miyagi Y, Takahashi S, Ito Y, Akiyama F, Ohno S, Iwase T. Accuracy of magnetic resonance imaging for predicting pathological complete response of breast cancer after neoadjuvant chemotherapy: association with breast cancer subtype. Springerplus 2016;5:152.

24. Hayashi Y, Takei H, Nozu S, Tochigi Y, Ichikawa A, Kobayashi N, Kurosumi M, Inoue K, Yoshida T, Nagai SE, Oba H, Tabei T, Horiguchi J, Takeyoshi I. Analysis of complete response by MRI following neoadjuvant chemotherapy predicts pathological tumor responses differently for molecular subtypes of breast cancer. Oncol Lett 2013;5:83-9.

25. Giuliano AE, Connolly JL, Edge SB, Mittendorf EA, Rugo HS, Solin LJ, Weaver DL, Winchester DJ, Hortobagyi GN. Breast Cancer-Major changes in the American Joint Committee on Cancer eighth edition cancer staging manual. CA Cancer J Clin 2017;67:290-303.

26. Fung F, Cornacchi SD, Vanniyasingam T, Dao D, Thabane L, Simunovic M, Hodgson N, O'Brien MA, Reid S, Heller B, Lovrics PJ. Predictors of 5-year local, regional, and distant recurrent events in a population-based cohort of breast cancer patients. Am J Surg 2017;213:418-25.

27. Goldhirsch A, Winer EP, Coates AS, Gelber RD, Piccart-Gebhart M, Thurlimann B, Senn HJ, Panel m. Personalizing the treatment of women with early breast cancer: highlights of the St Gallen International Expert Consensus on the Primary Therapy of Early Breast Cancer 2013. Ann Oncol 2013;24:2206-23.

28. Chen JH, Bahri S, Mehta RS, Carpenter PM, McLaren CE, Chen WP, Fwu PT, Hsiang DJ, Lane KT, Butler JA, $\mathrm{Su}$ MY. Impact of factors affecting the residual tumor size diagnosed by MRI following neoadjuvant chemotherapy in comparison to pathology. J Surg Oncol 2014;109:158-67.

29. Yu N, Leung VWY, Meterissian S. MRI Performance in Detecting pCR After Neoadjuvant Chemotherapy by Molecular Subtype of Breast Cancer. World J Surg 2019;43:2254-61.

30. Li H, Yao L, Jin P, Hu L, Li X, Guo T, Yang K. MRI and PET/CT for evaluation of the pathological response to neoadjuvant chemotherapy in breast cancer: A systematic review and meta-analysis. Breast 2018;40:106-15.

31. Dave RV, Millican-Slater R, Dodwell D, Horgan K, Sharma N. Neoadjuvant chemotherapy with MRI monitoring for breast cancer. Br J Surg 2017;104:1177-87.

32. Sener SF, Sargent RE, Lee C, Manchandia T, Le-Tran V, 
Olimpiadi Y, Zaremba N, Alabd A, Nelson M, Lang JE. MRI does not predict pathologic complete response after neoadjuvant chemotherapy for breast cancer. J Surg Oncol 2019;120:903-10.

33. Weber JJ, Jochelson MS, Eaton A, Zabor EC, Barrio AV, Gemignani ML, Pilewskie M, Van Zee KJ, Morrow M, El-Tamer M. MRI and Prediction of Pathologic Complete Response in the Breast and Axilla after Neoadjuvant Chemotherapy for Breast Cancer. J Am Coll Surg 2017;225:740-6.

34. Gampenrieder SP, Peer A, Weismann C, Meissnitzer M, Rinnerthaler G, Webhofer J, Westphal T, Riedmann M, Meissnitzer T, Egger H, Klaassen Federspiel F, Reitsamer R, Hauser-Kronberger C, Stering K, Hergan K, Mlineritsch B, Greil R. Radiologic complete response
(rCR) in contrast-enhanced magnetic resonance imaging (CE-MRI) after neoadjuvant chemotherapy for early breast cancer predicts recurrence-free survival but not pathologic complete response (pCR). Breast Cancer Res 2019;21:19.

35. Woodhams R, Kakita S, Hata H, Iwabuchi K, Kuranami M, Gautam S, Hatabu H, Kan S, Mountford C. Identification of residual breast carcinoma following neoadjuvant chemotherapy: diffusion-weighted imaging--comparison with contrast-enhanced MR imaging and pathologic findings. Radiology 2010;254:357-66.

36. Bufi E, Belli P, Di Matteo M, Terribile D, Franceschini G, Nardone L, Petrone G, Bonomo L. Effect of breast cancer phenotype on diagnostic performance of MRI in the prediction to response to neoadjuvant treatment. Eur J Radiol 2014;83:1631-8.

Cite this article as: Zhang $\mathrm{X}$, Wang $\mathrm{D}$, Liu Z, Wang Z, Li Q, $\mathrm{Xu} \mathrm{H}$, Zhang B, Liu T, Jin F. The diagnostic accuracy of magnetic resonance imaging in predicting pathologic complete response after neoadjuvant chemotherapy in patients with different molecular subtypes of breast cancer. Quant Imaging Med Surg 2020;10(1):197-210. doi: 10.21037/qims.2019.11.16 